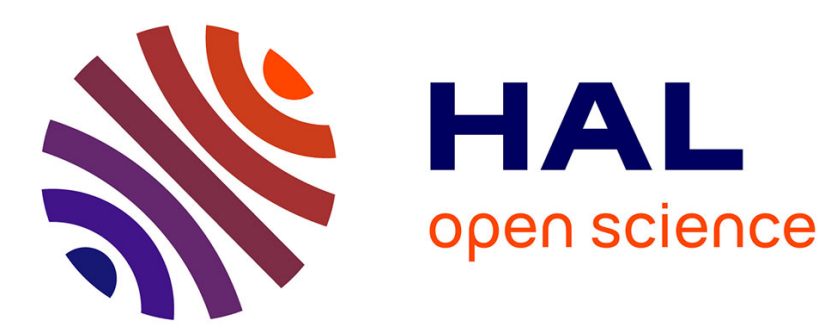

\title{
The Theta-dependence coefficient and an Almost Sure Limit Theorem for Random Iterative Models
}

\author{
R. Giuliano-Antonini, Michel Weber
}

\section{To cite this version:}

R. Giuliano-Antonini, Michel Weber. The Theta-dependence coefficient and an Almost Sure Limit Theorem for Random Iterative Models. Statistics and Probability Letters, 2009, 78 (5), pp.564. 10.1016/j.spl.2007.09.008 . hal-00594456

\section{HAL Id: hal-00594456 https://hal.science/hal-00594456}

Submitted on 20 May 2011

HAL is a multi-disciplinary open access archive for the deposit and dissemination of scientific research documents, whether they are published or not. The documents may come from teaching and research institutions in France or abroad, or from public or private research centers.
L'archive ouverte pluridisciplinaire HAL, est destinée au dépôt et à la diffusion de documents scientifiques de niveau recherche, publiés ou non, émanant des établissements d'enseignement et de recherche français ou étrangers, des laboratoires publics ou privés. 


\section{Author's Accepted Manuscript}

The Theta-dependence coefficient and an Almost Sure Limit Theorem for Random Iterative Models

R. Giuliano-Antonini, M. Weber

PII: $\quad$ S0167-7152(07)00288-X

DOI: $\quad$ doi:10.1016/j.spl.2007.09.008

Reference: $\quad$ STAPRO 4740

To appear in: $\quad$ Statistics \& Probability Letters

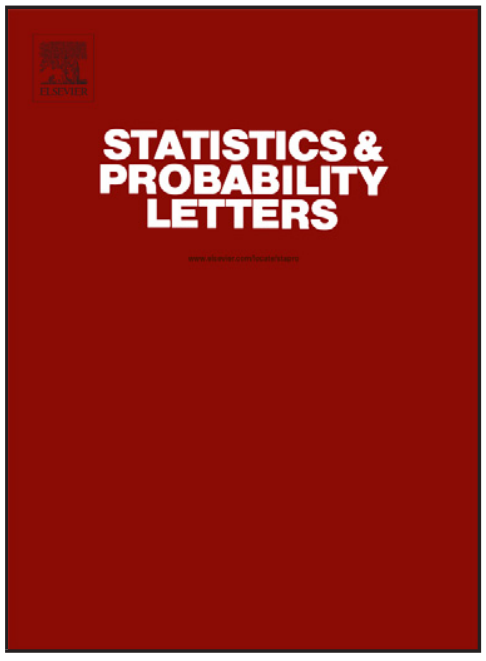

www.elsevier.com/locate/stapro

Received date: 15 December 2004

Revised date: $\quad 7$ July 2007

Accepted date: $\quad 7$ September 2007

Cite this article as: R. Giuliano-Antonini and M. Weber, The Theta-dependence coefficient and an Almost Sure Limit Theorem for Random Iterative Models, Statistics \& Probability Letters (2007), doi:10.1016/j.spl.2007.09.008

This is a PDF file of an unedited manuscript that has been accepted for publication. As a service to our customers we are providing this early version of the manuscript. The manuscript will undergo copyediting, typesetting, and review of the resulting galley proof before it is published in its final citable form. Please note that during the production process errors may be discovered which could affect the content, and all legal disclaimers that apply to the journal pertain. 


\title{
The Theta-dependence Coefficient and an Almost Sure Limit Theorem for Random Iterative Models
}

\author{
R. Giuliano-Antonini and M. WeBer
}

\begin{abstract}
We prove a weighted Almost Sure Limit Theorem in the setting of Random Iterative Models. This Theorem generalizes previous results obtained for sequences of normalized partial sums and some other classes of random sequences.
\end{abstract}

\section{Introduction}

Let $\left(S_{n}\right)$ be the partial sums of iid real valued random variables $\left(X_{n}\right)$ with mean 0 and variance 1 , defined on a probability space $(\Omega, \mathcal{A}, P)$. The classical Almost Sure Central Limit Theorem can be stated as follows: $P$-almost surely,

$$
\lim _{n \rightarrow \infty} \frac{1}{\log n} \sum_{k=1}^{n} \frac{1}{k}\left(1_{A}\left(\frac{S_{k}}{\sqrt{k}}\right)-P\left(\frac{S_{k}}{\sqrt{k}} \in A\right)\right)=0,
$$

for all Borel sets $A \subseteq \mathbb{R}$ such that $\lambda(\partial A)=0$. Here and in the sequel $\lambda$ denotes the Lebesgue measure on $(\mathbb{R}, \mathcal{B}(\mathbb{R}))$. For the proof, by a classical principle in the theory of pointwise Central Limit Theorem, (see [7], p. 202), it is enough to show that, for any bounded 1-Lipschitz function $f: \mathbb{R} \rightarrow \mathbb{R}$, almost surely one has

$$
\lim _{n \rightarrow \infty} \frac{1}{\log n} \sum_{k=1}^{n} \frac{1}{k}\left(f\left(\frac{S_{k}}{\sqrt{k}}\right)-\mathbf{E}\left[f\left(\frac{S_{k}}{\sqrt{k}}\right)\right]\right)=0,
$$

The proof of (1.1) relies on a suitable estimate of

$$
\operatorname{Cov}\left(f\left(\frac{S_{p}}{\sqrt{p}}\right), f\left(\frac{S_{q}}{\sqrt{q}}\right)\right)=\mathbf{E}\left[f\left(\frac{S_{p}}{\sqrt{p}}\right) f\left(\frac{S_{q}}{\sqrt{q}}\right)\right]-\mathbf{E}\left[f\left(\frac{S_{p}}{\sqrt{p}}\right)\right] \mathbf{E}\left[f\left(\frac{S_{q}}{\sqrt{q}}\right)\right],
$$

for $p \leq q$ integers. Typically such a kind of estimate looks as

$$
\left|\operatorname{Cov}\left(f\left(\frac{S_{p}}{\sqrt{p}}\right), f\left(\frac{S_{q}}{\sqrt{q}}\right)\right)\right| \leq \text { const } \sqrt{\frac{p}{q}}
$$

(see for instance [7], Lemma p. 203), and it is easy to prove (see Lemma (6.1) of the present paper) that we can get it from an analogous one for

$$
\left\|\mathbf{E}\left[f\left(\frac{S_{q}}{\sqrt{q}}\right) \mid \frac{S_{p}}{\sqrt{p}}\right]-\mathbf{E}\left[f\left(\frac{S_{q}}{\sqrt{q}}\right)\right]\right\|_{1},
$$

where $\|\cdot\|_{1}$ denotes the $L^{1}(\Omega, \mathcal{A}, P)$ - norm. Note that the two random variables $\frac{S_{n+1}}{\sqrt{n+1}}, \frac{S_{n}}{\sqrt{n}}$ are linked by the iterative equation

$$
\frac{S_{n+1}}{\sqrt{n+1}}=\sqrt{\frac{n}{n+1}} \frac{S_{n}}{\sqrt{n}}+\frac{X_{n+1}}{\sqrt{n+1}}=\sqrt{\frac{n}{n+1}} \frac{S_{n}}{\sqrt{n}}+V_{n+1},
$$

Date: on April 23, 2007. AMS Subject Classification 2000: Primary 60F15, Secondary 60G12.

Keywords: $\vartheta$-dependence coefficient, Almost Sure Limit Theorem, Random Iterative Model, Lipschitzian Model, Autoregressive Model, Quasi Orthogonal System.

This work is partially supported by the M.I.U.R. Italy. 
where $V_{n+1}=\frac{X_{n+1}}{\sqrt{n+1}}$. In this paper we consider a system of random $d$-dimensional vectors $\left(Z_{n}\right)$ defined on a probability space $(\Omega, \mathcal{A}, P)$ by a recursive relation $Z_{n+1}=F_{n+1}\left(Z_{n}, V_{n+1}\right)$ and, under suitable assumptions, we prove an estimate for

$$
\vartheta\left(Z_{q}, Z_{p}\right) \doteq \sup _{f \in \mathcal{L}_{1}}\left\|\mathbf{E}\left[f\left(Z_{q}\right) \mid Z_{p}\right]-\mathbf{E}\left[f\left(Z_{q}\right)\right]\right\|_{1},
$$

where $\mathcal{L}_{1}$ denotes the set of bounded 1-Lipschitz functions $f: \mathbb{R}^{d} \rightarrow \mathbb{R}$. Such an estimate (which will be in terms of the sequence $\left(F_{n}\right)$ ) allows us to prove an Almost Sure Limit Theorem (ASLT from now on) for the sequence $\left(Z_{n}\right)$; this result enlarges the classical Almost Sure Central Limit Theorem since it concerns "general" weights and not "logarithmic" weights only; some new particular cases are pointed out in (5.17).

Previous results in this direction can be found for instance in [1] and [8]; with respect to the results of [1], our Theorem enlightens the fact that weak theorems are not necessary in order to obtain Almost Sure Limit Theorems; moreover, condition (1.7) of [1] is more difficult to be checked than our condition (2.11) (see Remark (2.12)); last, our examples (4.1) (in particular (4.1)(iii) and 4.1(iv) ), (4.4), (4.5) are new (see also Examples (5.17)). On the other hand, with respect to [8] our Theorem is wider in that it concerns a general "Iterative Model" $\left(Z_{n}\right)$ (see definition (2.1)), and not only a sequence of normalized partial sums $\left(S_{n} / \sqrt{n}\right)$.

We stress the fact that the setting of iterative models considered here is rather large: see section 4 for some illuminating examples. The coefficient $\vartheta\left(Z_{q}, Z_{p}\right)$ defined in (1.3) is clearly a measure of the dependence of $Z_{q}$ and $Z_{p}$. It is known in the literature (see [2] for details an the references therein); we shall call it coefficient of $\vartheta$-dependence; in sections 3 and 4 we show how to calculate it in some cases. Another frequently used measure of dependence between random variables is the Rosenblatt coefficient (see section 6 for its definition); in the same section 6 we present a typical situation in which the Rosenblatt coefficient can be obtained from the $\vartheta$-coefficient.

Acknowledgement. The authors wish to thank Prof. L. Pratelli for helpful suggestions and an unknown referee for having pointed out an error in the proof of Theorem (2.9).

\section{Main results}

On the probability space $(\Omega, \mathcal{A}, P)$ we are given a filtration $\left(\mathcal{A}_{n}\right)$. Following [4], p. 183 we introduce the concept of Iterative Lipschitzian Model:

(2.1) Definition. An Iterative Lipschitzian Model adapted to $\left(\mathcal{A}_{n}\right)$ is a sequence $\left(Z_{n}\right)$ of random $d$-dimensional vectors such that, for every $n \in \mathbb{N}, Z_{n}$ is $\mathcal{A}_{n}$-measurable and

$$
Z_{n+1}=F_{n+1}\left(Z_{n}, V_{n+1}\right)
$$

where,

(i) for every $n \in \mathbb{N}, F_{n}$ is a measurable function from $\mathbb{R}^{d} \times \Gamma$ to $\mathbb{R}^{d}$ (where $(\Gamma, \mathcal{G}$ ) is a measurable space), $\alpha_{n^{-}}$Lipschitzian in its first argument, independent on the second, $i$. e., for each $z_{1}, z_{2} \in \mathbb{R}^{d}$, $v \in \Gamma$

$$
\left|F_{n}\left(z_{1}, v\right)-F_{n}\left(z_{2}, v\right)\right| \leq \alpha_{n}\left|z_{1}-z_{2}\right| .
$$

Here and in the sequel $|\cdot|$ denotes the euclidean norm in $\mathbb{R}^{d}$.

(ii) $\left(V_{n}\right)$ is a sequence of random variables with values in $(\Gamma, \mathcal{G})$, and $V_{n+1}$ is independent of $\mathcal{A}_{n}$ for every $n \in \mathbb{N}$.

Our first result is the following.

(2.3) Theorem. Assume that $\sup _{n} \mathbf{E}\left[\left|Z_{n}\right|\right]=C<+\infty$. Then, for $p<q$ integers, we have

$$
\vartheta\left(Z_{q}, Z_{p}\right) \leq 2 C \alpha_{p+1} \times \cdots \times \alpha_{q} .
$$

Write

$$
g(k)=\prod_{h=1}^{k} \frac{1}{\alpha_{h}}
$$


Then (2.4) can be written as

$$
\vartheta\left(Z_{q}, Z_{p}\right) \leq 2 C \frac{g(p)}{g(q)} .
$$

The proof of Theorem (2.3) is in Section 3. Before stating our second result (the $A S L T$ for the sequence $\left.\left(Z_{n}\right)\right)$ we must recall some preliminary notions and make some remarks.

(i) Let $(T, \mathcal{C}, \tau)$ be some probability space and consider a sequence $\left(f_{n}\right)$ of elements of $L^{2}(\tau)$. Let $a_{h, k}=\int f_{h} f_{k} d \tau$. A system of functions $\left(f_{n}\right)$ such that the quadratic form defined on $\ell_{2}$ by $\left(x_{n}\right) \mapsto$ $\sum_{h, k} a_{h, k} x_{h} x_{k}$ is bounded, is said quasi orthogonal. Say also that a sequence $c=\left(c_{n}\right) \in \ell_{2}$ is universal when the series $\sum_{k} c_{k} \psi_{k}$ converges almost everywhere for every orthonormal system of functions $\left(\psi_{n}\right)$. According to Schur's Theorem ([9], pag. 56), if $c$ is universal, then the series $\sum_{k} c_{k} f_{k}$ converges almost everywhere for any quasi-orthogonal system of functions $\left(f_{n}\right)$.

(ii) Assume that $\left(Z_{n}\right)$ is an iterative Lipschitzian model, such that

$$
\begin{gathered}
\alpha_{n}<1 \quad \forall n \geq 2, \quad \sum_{k=2}^{\infty} \log \alpha_{k}=-\infty \\
\liminf _{n \rightarrow \infty} \alpha_{n}>0 .
\end{gathered}
$$

Condition (2.7) amounts clearly to assuming that $g$, defined in (2.5), is strictly increasing to $+\infty$, so that we can suppose that it is defined on $[1,+\infty)$ and strictly increasing to $+\infty$. On the other hand, condition (2.8) is equivalent to $\limsup _{x \rightarrow \infty} g(x+1) / g(x)<+\infty$. We can now state our result.

(2.9) TheOREM. (ASLT for iterative models). Let $\left(Z_{n}\right)$ be an iterative Lipschitzian model such that $\sup _{n} \mathbf{E}\left[\left|Z_{n}\right|\right]=C<+\infty$. Let $\varphi:[1,+\infty) \rightarrow \mathbb{R}^{+}$be a strictly increasing function with

$$
\lim _{x \rightarrow+\infty} \varphi(x)=+\infty
$$

and for which there exists a constant $\beta>0$ such that

$$
\varphi(x+1) \leq \varphi(x)+\beta \quad \forall x \in \mathbb{R}^{+} .
$$

Assume moreover that the function $g$ defined in (2.5) can be extended to $[1,+\infty)$ and that the composed function $G=g \circ \varphi^{-1}$ verifies the condition

$$
\sup _{n}\left(\sum_{k \leq n} \frac{G(k)}{G(n)}+\sum_{k>n} \frac{G(n)}{G(k)}\right)<+\infty .
$$

Let $\mathcal{E}=\left\{A \in \mathcal{B}\left(\mathbb{R}^{d}\right), \lambda(\partial A)=0\right\}$. Then, for every $A \in \mathcal{E}$,

(a) for every decreasing sequence $\left(c_{n}\right)$ satisfying the conditions

$$
\lim _{n \rightarrow \infty}\left(c_{n-1}-c_{n}\right) n=0, \quad \liminf _{n \rightarrow \infty}\left(c_{n+1} / c_{n}\right)>0, \quad \sum_{n} c_{n}^{2}(\log n)^{2}<\infty,
$$

almost surely we have

$$
\lim _{n \rightarrow \infty} c_{[\varphi(n)]} \sum_{k=1}^{n}(\varphi(k+1)-\varphi(k))\left(1_{A}\left(Z_{k}\right)-P\left(Z_{k} \in A\right)\right)=0 ;
$$

(b) almost surely we have

$$
\lim _{n \rightarrow \infty} \frac{\sum_{k=1}^{n}(\varphi(k+1)-\varphi(k))\left(1_{A}\left(Z_{k}\right)-P\left(Z_{k} \in A\right)\right)}{\varphi(n)}=0 .
$$

(2.12) REMARK. If the iterative model $\left(Z_{n}\right)$ satisfies (2.7) and (2.8), a function $\varphi$ verifying the two conditions (2.10) and (2.11) is easily found: in fact we can take, for instance

$$
\varphi(x)=\log g(x) .
$$

The proofs of Theorem (2.9) and Remark (2.12) will be given in Section 5. 


\section{The coefficient of $\vartheta$-dependence and the proof of Theorem (2.3)}

Theorem (2.3) follows easily from a general result (Proposition (3.2)), which we state and prove in this section. Let $T$ and $S$ be two $d$-dimensional random vectors defined on a probabilty space $(\Omega, \mathcal{A}, P)$ and assume that $\mathbf{E}[|T|]<+\infty$; let $\mathcal{L}_{1}$ be the set of bounded functions $f: \mathbb{R}^{d} \rightarrow \mathbb{R}$ which are Lipschitzian of constant 1 .

(3.1) Definition. For $T$ and $S$ as above, we define the $\vartheta$-coefficient of dependence as

$$
\vartheta(T, S) \doteq \sup _{f \in \mathcal{L}_{1}}|| \mathbf{E}[f(T) \mid S]-\mathbf{E}[f(T)] \|_{1}
$$

where $\|\cdot\|_{1}$ denotes the norm in $L^{1}(\Omega, \mathcal{A}, P)$.

We are interested in the following situation: assume that $T=\phi(S, V)$, where $V$ is a random variable defined on $(\Omega, \mathcal{A}, P)$ with values in a measurable space $(\Gamma, \mathcal{G})$, independent on $S$, and $\phi: \mathbb{R}^{d} \times \Gamma \rightarrow \mathbb{R}^{d}$ is a measurable function $\beta$-Lipschitzian in its first argument, independent on the second, i. e. for every $s_{1}, s_{2} \in \mathbb{R}^{d}$ and $v \in \Gamma$ we have

$$
\left|\phi\left(s_{1}, v\right)-\phi\left(s_{2}, v\right)\right| \leq \beta\left|s_{1}-s_{2}\right| .
$$

We prove our general result:

(3.2) Proposition. Assume that $S$ is integrable. Then

$$
\vartheta(T, S) \leq 2 \beta \mathbf{E}[|S|]
$$

Proof. It is easy to see that, for every $f \in \mathcal{L}_{1}$ we have

$$
\mathbf{E}[f(T) \mid S]=\int f \circ \phi(S, v) d \mu_{V}(v),
$$

where $\mu_{V}$ denotes the law of $V$. Hence

$$
\vartheta(T, S)=\sup _{f \in \mathcal{L}_{1}}\left\|\int f \circ \phi(S, v) d \mu_{V}(v)-\mathbf{E}\left[\int f \circ \phi(S, v) d \mu_{V}(v)\right]\right\|_{1} .
$$

The relation

$$
\left|\int f \circ \phi\left(s_{1}, v\right) d \mu_{V}(v)-\int f \circ \phi\left(s_{2}, v\right) d \mu_{V}(v)\right| \leq \int\left|\phi\left(s_{1}, v\right)-\phi\left(s_{2}, v\right)\right| d \mu_{V}(v) \leq \beta\left|s_{1}-s_{2}\right|
$$

shows that the function $g: s \mapsto \int f \circ \phi(s, v) d \mu_{V}(v)$ is $\beta$-Lipschitzian, hence

$$
\vartheta(T, S) \leq \beta \sup _{g \in \mathcal{L}_{1}}\|g(S)-\mathbf{E}[g(S)]\|_{1}=\beta \sup \left\{\int|g| d \mu_{S}, g \in \mathcal{L}_{1}, \int g d \mu_{S}=0\right\},
$$

where $\mu_{S}$ is the law of $S$. The statement of the proposition follows from a simple lemma.

(3.3) Lemma. Let $\mu$ be a probability measure on $\mathbb{R}^{d}$, with $\int|x| d \mu(x)<\infty$. Then

$$
\sup \left\{\int|g| d \mu, g \in \mathcal{L}_{1}, \int g d \mu=0\right\} \leq 2 \int|x| d \mu(x) .
$$

Proof. Let $g \in \mathcal{L}_{1}$ with $\int g d \mu=0$. Then

$$
|g(0)|=\left|\int(g(x)-g(0)) d \mu(x)\right| \leq \int|x| d \mu(x),
$$

hence $\int|g(x)| d \mu(x) \leq \int|g(x)-g(0)| d \mu(x)+|g(0)| \leq 2 \int|x| d \mu(x)$, and the lemma is proved. 
(3.5) Remark. Though not relevant in this context, note that in (3.3) it is possible to find a better estimate; in fact, one can replace the vector 0 (used in (3.4)) with any vector $x_{0} \in \mathbb{R}^{d}$ and then take the infimum with respect to $x_{0}$, so getting the bound

$$
2 \inf _{x_{0} \in \mathbb{R}^{d}} \int\left|x-x_{0}\right| d \mu(x) .
$$

We can now deduce Theorem (2.3) from Proposition (3.2); in fact from relation (2.2) it is easily seen by induction that

$$
Z_{q}=\phi_{p, q}\left(Z_{p}, V_{p+1}, \ldots, V_{q}\right)
$$

where $\phi_{q, p}$ is some function, $\left(\alpha_{p+1} \times \cdots \times \alpha_{q}\right)$-Lipschitzian in the first argument and $\left(V_{p+1}, \ldots, V_{q}\right)$ is a random variable with values in $\left(\Gamma^{q-p}, \mathcal{G}^{q-p}\right)$, independent on $Z_{p}$ (of course $\phi_{p+1, p}=F_{p+1}$ for every $p$ ).

(3.6) REMARK. We point out the important particular case (to be encountered later, see Example (4.5)) in which $\alpha_{n} \leq \alpha$ (constant) for every $n$. In this case we find

$$
\vartheta\left(Z_{q}, Z_{p}\right) \leq 2 C \alpha^{q-p} .
$$

\section{Some examples}

In this section we give some relevant examples of iterative Lipschitzian models. All the involved sequences of random variables are tacitly assumed to be defined on the basic probability space $(\Omega, \mathcal{A}, P)$.

(4.1) EXAMPLE. Let $\left(X_{n}\right)$ be a sequence of independent r. v.'s and $\left(\gamma_{n}\right)$ a sequence of positive numbers. Put $S_{n}=\sum_{k=1}^{n} \gamma_{k} X_{k}$ and assume that, for every $n, S_{n} \in L^{1}(\Omega, \mathcal{A}, P)$. Suppose that there exists a sequence $\left(a_{n}\right)$ of real numbers such that

$$
\sup _{n} \mathbf{E}\left[\left|\frac{S_{n}}{a_{n}}\right|\right]=C<+\infty
$$

For every integer $n$ define $Z_{n}=\frac{S_{n}}{a_{n}}$ and consider the maps $F_{n}(z, v)=\frac{a_{n-1}}{a_{n}} z+v$. Observe that

$$
Z_{n+1}=F_{n+1}\left(Z_{n}, V_{n+1}\right), \quad \text { with } \quad V_{n+1}=\frac{\gamma_{n+1} X_{n+1}}{a_{n}}
$$

Theorem (2.3) gives, for $p<q$,

$$
\vartheta\left(Z_{q}, Z_{p}\right) \leq 2 C \frac{a_{p}}{a_{q}}
$$

We are in the above setting if for instance

(i) $\sup _{n} E\left[\left|X_{n}\right|\right]<+\infty$.

In this case we can take $a_{n}=\sum_{k=1}^{n} \gamma_{k}$, as it is easily seen.

(ii) If $\sigma_{n}^{2}=\mathbf{E}\left[X_{n}^{2}\right]<+\infty$ for every $n$, another suitable choice for $\left(a_{n}\right)$ is $a_{n}=\left(\sum_{k=1}^{n} \sigma_{k}^{2} \gamma_{k}^{2}\right)^{1 / 2}$.

(iii) $\gamma_{n}=1$ for every $n,\left(X_{n}\right)$ are independent identically distributed and their common distribution belongs to the domain of attraction of a stable distribution $\Phi$ with exponent $\alpha \in(1,2]$. This means that there exist two sequences of numbers $\left(a_{n}\right)$ and $\left(b_{n}\right)$ such that the distribution of $\frac{S_{n}}{a_{n}}-b_{n}=Z_{n}-b_{n}$ tends to $\Phi$. In this case it is known (see [6], lemma 2.3) that $\mathbf{E}\left[\left|S_{n}\right|\right] \leq C a_{n}$ for a suitable constant $C$.

(iv) Let $p>1$, and consider the class $\mathcal{F}_{p}$ of distribution functions verifying

$$
\left(F(-x) \vee(1-F(x))=\mathcal{O}\left(x^{-p}\right) \quad x \rightarrow+\infty .\right.
$$

Let again $\gamma_{n}=1$ for every $n,\left(X_{n}\right)$ be independent identically distributed with their common law belonging to $\mathcal{F}_{p}$. According to part b) of Lemma 2.2 in [6], if $F \in \mathcal{F}_{p}, 1<p<2$, then $\mathbf{E}\left|S_{n}\right| \leq C_{p} n^{1 / p}$. And if $p=2$ and $\mathbf{E} X^{2}=\infty$, then $\mathbf{E}\left|S_{n}\right| \leq C\left(n \mathbf{E} X^{2} \mathbf{1}_{\left\{|X| \leq n^{1 / 2}\right\}}\right)^{1 / 2}$, which gives other examples of the same kind as above. 
(4.3) EXAMPLE. Let $\left(X_{n}\right)$ be a sequence of independent random variables with $\sup _{n} \mathbf{E}\left[\left|X_{n}\right|\right]=C<+\infty$. Put $M_{n}=\max \left(X_{1}, \ldots, X_{n}\right), Z_{n}=\frac{M_{n}}{n}$. Then $\mathbf{E}\left[\left|Z_{n}\right|\right] \leq \frac{\sum_{k=1}^{n} \mathbf{E}\left[\left|X_{k}\right|\right]}{n} \leq C<+\infty$.

Put $F_{n}(z, v)=\max \left(\frac{n-1}{n} z, \frac{v}{n}\right)$. Then $F_{n}$ is $((n-1) / n)$-Lipschitzian and we have $Z_{n+1}=F_{n+1}\left(Z_{n}, V_{n+1}\right)$, with $V_{n+1}=\frac{X_{n+1}}{n+1}$. Theorem (2.3) applies and we get

$$
\vartheta\left(Z_{q}, Z_{p}\right) \leq C \frac{p}{q}
$$

(4.4) ExAmple. Let $X_{n}$ be a sequence of independent identically distributed complex-valued random variables and let $f_{1}, f_{2}, \ldots$ be complex-valued functions defined on some metric space (T, $\left.\mathbf{d}\right)$, and form the quantities

$$
Z_{n}(t)=\frac{\sum_{k=1}^{N_{n}} \Re\left(X_{k} \overline{f_{k}(t)}\right)}{B_{n}(t)}, \quad B_{n}(t)=\left\|\sum_{k=1}^{N_{n}} \Re\left(X_{k} \overline{f_{k}(t)}\right)\right\|_{2, P} .
$$

where $\left(N_{n}\right)$ is some given sequence of integers. Here $t$ is fixed and we write more simply $Z_{n}:=Z_{n}(t)$, $B_{n}:=B_{n}(t)$. It is clear that

$$
Z_{n+1}=\frac{B_{n}}{B_{n+1}} Z_{n}+V_{n+1}, \quad V_{n+1}=\frac{\sum_{k=N_{n}+1}^{N_{n+1}} \Re\left(X_{k} \overline{f_{k}(t)}\right)}{B_{n+1}}
$$

Define $F_{n+1}(x, y)=\frac{B_{n}}{B_{n+1}} x+y$. Then we have $Z_{n+1}=F_{n+1}\left(Z_{n}, V_{n+1}\right)$. According to Definition (2.1), $\left(Z_{n}\right)$ is an iterative Lipschitzian model. Now by construction $\sup _{n} \mathbf{E}\left|Z_{n}\right|=C<\infty$. Indeed, by CauchySchwarz inequality, $\mathbf{E}\left[\left|Z_{n}(t)\right|\right]=\mathbf{E}\left[\left|\sum_{k=1}^{N_{n}} \Re\left(X_{k} \overline{f_{k}(t)}\right)\right|\right] / B_{n}(t) \leq 1$ so that Theorem (2.3) applies in force with $C=1$, and we get for $p \leq q$ :

$$
\vartheta\left(Z_{q}, Z_{p}\right) \leq 2 \frac{B_{p}}{B_{q}}
$$

(4.5) ExAmple. Let $\left(M_{n}\right)$ be a sequence of square $d \times d$ matrices with elements in $\mathbb{R}$, and let $\left\|M_{n}\right\|$ be the sequence of their norms. We assume that $\rho \doteq \sup _{n}|| M_{n} \|<1$. We consider the autoregressive model

$$
Z_{n+1}=F_{n+1}\left(Z_{n}, \varepsilon_{n+1}\right)=M_{n} Z_{n}+\varepsilon_{n+1}
$$

with initial state $Z_{0}$, where $\varepsilon=\left(\varepsilon_{n}\right)$ is a noise independent on $Z_{0}$. We assume that $\left|Z_{0}\right|$ is integrable. Moreover the $\left(\varepsilon_{n}\right)$ are independent and $\sup _{n} \mathbf{E}\left[\left|\varepsilon_{n}\right|\right]=C<+\infty$. By induction one sees easily that $Z_{n}$ and $\varepsilon_{n+1}$ are independent. Put

$$
B_{j}= \begin{cases}I_{d} & \text { for } j=0 \\ \prod_{k=n-j+1}^{n} M_{k} & \text { for } 1 \leq j \leq n\end{cases}
$$

Then $Z_{n}$ can be written in the closed form $Z_{n}=B_{n} Z_{0}+\sum_{k=1}^{n} B_{n-k} \varepsilon_{k}$. We have

$$
\sup _{n} \mathbf{E}\left[\left|X_{n}\right|\right] \leq \rho^{n} \mathbf{E}\left[\left|Z_{0}\right|\right]+C \sum_{k=1}^{n} \rho^{n-k} \leq \mathbf{E}\left[\left|Z_{0}\right|\right]+C \frac{1}{1-\rho}=C_{1} .
$$

Since $F_{n}$ is $\left\|M_{n}\right\|$-Lipschitzian, we deduce from Theorem (2.3) that

$$
\vartheta\left(Z_{q}, Z_{p}\right) \leq C_{1} \rho^{q-p}, \quad q \geq p
$$

\section{Proof of Theorem (2.9)}

As we saw in the Introduction, in order to prove (2.9) it is enough to prove an analogous result by substituting $1_{A}$ with any bounded function $f \in \mathcal{L}_{1}$. So, fix $f \in \mathcal{L}_{1}$. Throughout the present section we put, for every integer $n$

$$
Y_{n}=f\left(Z_{n}\right)-\mathbf{E}\left[f\left(Z_{n}\right)\right]
$$


Assertion (b) of Theorem (2.9) easily follows easily from assertion (a): take the sequence $\left(c_{n}\right)$ defined by $c_{n}=\frac{1}{\sqrt{n} \log ^{2} n}$, and observe that $\left(c_{n}\right)$ is universal by Rademacher-Menchov Theorem, (asserting that a sequence $\left(c_{n}\right)$ is universal if $\left.\sum_{n} c_{n}^{2} \log ^{2} n<+\infty\right)$. Now we have

$$
\frac{\left|\sum_{k=1}^{n}(\varphi(k+1)-\varphi(k)) Y_{k}\right|}{\varphi(n)} \leq \frac{\log ^{2} \varphi(n)}{\sqrt{\varphi(n)}}\left|c_{[\varphi(n)]} \sum_{k=1}^{n}(\varphi(k+1)-\varphi(k)) Y_{k}\right|
$$

and the second term of the above inequality tends to 0 by assertion (a); hence we prove assertion (a). With no loss of generality we can assume $\beta=1$ in $(2.10)$. This is plain if $\beta \leq 1$ : if $\beta>1$, we prove first the result for the function $\tilde{\varphi} \doteq \beta^{-1} \varphi$; in order to get the desired conclusion for the function $\varphi$ also, we need only to observe that

$$
c_{[\varphi(n)]} \leq c_{\left[\varphi(n) \beta^{-1}\right]}
$$

We now remark that the relation

$$
\varphi(x+1) \leq \varphi(x)+1 \quad \forall x \in \mathbb{R}^{+}
$$

implies (in fact, is equivalent to)

$$
\varphi^{-1}(x)+1 \leq \varphi^{-1}(x+1) \quad \forall x \in \mathbb{R}^{+} .
$$

Put $\psi(k)=\left[\varphi^{-1}(k)\right]$ for every integer $k$. From (5.1) we get also

$$
\psi(k)+1 \leq \psi(k+1) \quad \forall k \in \mathbb{N}^{*}
$$

Relation (5.2) implies in turn

$$
\varphi(\psi(k))-\varphi(\psi(k-1)) \leq \varphi(\psi(k))-\varphi(\psi(k-2)+1) \leq \varphi\left(\varphi^{-1}(k)\right)-\varphi\left(\varphi^{-1}(k-2)\right)=2 .
$$

We now need two Lemmas.

(5.4) Lemma. Let $p \leq q$ be two integers. Then, for every $g \in \mathcal{L}_{1}$ the following inequality holds

$$
\left|\operatorname{Cov}\left(g\left(Z_{p}\right), g\left(Z_{q}\right)\right)\right| \leq \sup |g| \vartheta\left(Z_{q}, Z_{p}\right)
$$

The proof of the above Lemma is quite similar to the proof of Lemma (6.1), and is omitted.

(5.5) Remark. Note that the functions $g_{1}$ and $g_{2}$ defined respectively as $g_{1}(x)=\left(f(x)-\mathbf{E}\left[f\left(Z_{n}\right)\right]\right)^{+}$ and $g_{2}(x)=\left(f(x)-\mathbf{E}\left[f\left(Z_{n}\right)\right]\right)^{-}$belong to $\mathcal{L}_{1} ;$ moreover $\sup \left|g_{i}\right| \leq 2 \sup |f|(i=1,2)$. Thus, Lemma (5.4) implies that

$$
\left|\operatorname{Cov}\left(Y_{p}^{+}, Y_{q}^{+}\right)\right| \leq 2 \sup |f| \vartheta\left(Z_{q}, Z_{p}\right)
$$

and analogously

$$
\left|\operatorname{Cov}\left(Y_{p}^{-}, Y_{q}^{-}\right)\right| \leq 2 \sup |f| \vartheta\left(Z_{q}, Z_{p}\right)
$$

We can now pass to the proof of point (a) of (2.9). For every $n$ put

$$
U_{n}^{+}=c_{n} \sum_{k=1}^{n} Y_{\psi(k)}^{+}, \quad V_{n}^{+}=c_{n} \sum_{k=1}^{\psi(n)-1}(\varphi(k+1)-\varphi(k)) Y_{k}^{+}, \quad T_{n}^{+}=c_{[\varphi(n)]} \sum_{k=1}^{n}(\varphi(k+1)-\varphi(k)) Y_{k}^{+}
$$

and 


$$
U_{n}^{-}=c_{n} \sum_{k=1}^{n} Y_{\psi(k)}^{-}, \quad V_{n}^{-}=c_{n} \sum_{k=1}^{\psi(n)-1}(\varphi(k+1)-\varphi(k)) Y_{k}^{-}, \quad T_{n}^{-}=c_{[\varphi(n)]} \sum_{k=1}^{n}(\varphi(k+1)-\varphi(k)) Y_{k}^{-}
$$

(5.8) Lemma. We have, P-almost surely

$$
\lim _{n \rightarrow \infty}\left(U_{n}^{+}-\mathbf{E}\left[U_{n}^{+}\right]\right)=0 ; \lim _{n \rightarrow \infty}\left(U_{n}^{-}-\mathbf{E}\left[U_{n}^{-}\right]\right)=0 .
$$

Proof. We prove the first relation. Note that $U_{n}^{+}-\mathbf{E}\left[U_{n}^{+}\right]=c_{n} \sum_{k=1}^{n}\left(Y_{\psi(k)}^{+}-\mathbf{E}\left[Y_{\psi(k)}^{+}\right]\right)$. For $h \leq k$, by (5.6), (2.6) and (5.2),

$$
\left|\operatorname{Cov}\left(Y_{\psi(h)}^{+}, Y_{\psi(k)}^{+}\right)\right| \leq 2 C \frac{G(h)}{G(k-1)} .
$$

Now condition (2.11) assures that the sequence $\left(Y_{\psi(n)}^{+}-\mathbf{E}\left[Y_{\psi(n)}^{+}\right]\right)$is quasi orthogonal by lemma 7.4.3 p. 139 of [11]. The result thus follows from Kronecker's Lemma.

The second relation has identical proof (use (5.7) instead of (5.6)).

(5.9) Lemma. We have, P-almost surely

$$
\lim _{n \rightarrow \infty}\left(V_{n}^{+}-\mathbf{E}\left[V_{n}^{+}\right]\right)=0 ; \quad \lim _{n \rightarrow \infty}\left(V_{n}^{-}-\mathbf{E}\left[V_{n}^{-}\right]\right)=0
$$

Proof. Once more we prove only the first relation above, since the second one has identical proof.

By Lemma (5.8), it is enough to prove that the sequence $\left\{\left(U_{n}^{+}-V_{n}^{+}\right)-\mathbf{E}\left[U_{n}^{+}-V_{n}^{+}\right]\right\}_{n}$ converges to 0 almost surely. Now

$$
\begin{aligned}
U_{n}^{+}-V_{n}^{+} & =c_{n}\left(\sum_{k=1}^{n} Y_{\psi(k)}^{+}-\sum_{k=1}^{\psi(n)-1}(\varphi(k+1)-\varphi(k)) Y_{k}^{+}\right) \\
& =c_{n}\left(\sum_{k=1}^{n} Y_{\psi(k)}^{+}-\sum_{k=1}^{n} \sum_{j=\psi(k-1)}^{\psi(k)-1}(\varphi(j+1)-\varphi(j)) Y_{j}^{+}\right)=c_{n} \sum_{k=1}^{n} \sum_{j=\psi(k-1)}^{\psi(k)} \delta_{j} Y_{j}^{+},
\end{aligned}
$$

where we put

$$
\delta_{j}= \begin{cases}\varphi(j)-\varphi(j+1) & \text { for } \psi(k-1) \leq j \leq \psi(k)-1 \\ 1 & \text { for } j=\psi(k)\end{cases}
$$

We have easily, by (5.3)

$$
\sum_{j=\psi(k-1)}^{\psi(k)}\left|\delta_{j}\right|=1+\sum_{j=\psi(k-1)}^{\psi(k)-1}(\varphi(j+1)-\varphi(j))=1+[\varphi(\psi(k))-\varphi(\psi(k-1))] \leq 3 .
$$

Put now $R_{k}=\sum_{j=\psi(k-1)}^{\psi(k)} \delta_{j} Y_{j}^{+}$. We have to prove that $\lim _{n \rightarrow \infty} c_{n} \sum_{k=1}^{n}\left(R_{k}-\mathbf{E}\left[R_{k}\right]\right)=0$ almost surely.

We need a bound for $\operatorname{Cov}\left(R_{h}, R_{k}\right)$. We have

$$
\operatorname{Cov}\left(R_{h}, R_{k}\right)=\sum_{i=\psi(h-1)}^{\psi(h)-1} \sum_{j=\psi(k-1)}^{\psi(k)-1} \delta_{i} \delta_{j} \operatorname{Cov}\left(Y_{i}^{+}, Y_{j}^{+}\right)
$$

Now, for every $i, j$ with $\psi(h-1) \leq i \leq \psi(h), \psi(k-1) \leq j \leq \psi(k)$, we have, again by (5.6) and (2.6),

$$
\left|\operatorname{Cov}\left(Y_{i}^{+}, Y_{j}^{+}\right)\right| \leq C_{1} \frac{G(h)}{G(k-2)}
$$

Condition (2.11) and relation (5.10) assure that the sequence $\left(R_{n}\right)$ is quasi orthogonal, and we can now argue as in the proof of Lemma (5.8). 
It is now easily verified that for $\psi(r) \leq n \leq \psi(r+1)-1$ we have

$$
\frac{c_{r+1}}{c_{r}} V_{r}^{+} \leq T_{n}^{+} \leq \frac{c_{r-1}}{c_{r+1}} V_{r+1}^{+}, \quad \frac{c_{r+1}}{c_{r}} V_{r}^{-} \leq T_{n}^{-} \leq \frac{c_{r-1}}{c_{r+1}} V_{r+1}^{-}
$$

whence, putting

$$
T_{n}=T_{n}^{+}-T_{n}^{-}=c_{[\varphi(n)]} \sum_{k=1}^{n}(\varphi(k+1)-\varphi(k)) Y_{k},
$$

we get

$$
\frac{c_{r+1}}{c_{r}} V_{r}^{+}-\frac{c_{r-1}}{c_{r+1}} V_{r+1}^{-} \leq T_{n} \leq \frac{c_{r-1}}{c_{r+1}} V_{r+1}^{+}-\frac{c_{r+1}}{c_{r}} V_{r}^{-} .
$$

In view of (5.11), everything is now reduced to prove that, $P$-a.s

$$
\lim _{r \rightarrow \infty} \frac{c_{r+1}}{c_{r}} V_{r}^{+}-\frac{c_{r-1}}{c_{r+1}} V_{r+1}^{-}=0 ; \quad \lim _{r \rightarrow \infty} \frac{c_{r-1}}{c_{r+1}} V_{r+1}^{+}-\frac{c_{r+1}}{c_{r}} V_{r}^{-}=0 .
$$

We prove the second one of relations (5.12) (the first one is identical). We have

$$
\frac{c_{r-1}}{c_{r+1}} V_{r+1}^{+}-\frac{c_{r+1}}{c_{r}} V_{r}^{-}=\left\{\frac{c_{r-1}}{c_{r+1}}\left(V_{r+1}^{+}-\mathbf{E}\left[V_{r+1}^{+}\right]\right)\right\}+\left\{-\frac{c_{r+1}}{c_{r}}\left(V_{r}^{-}-\mathbf{E}\left[V_{r}^{-}\right]\right)\right\}+\left\{\frac{c_{r-1}}{c_{r+1}} \mathbf{E}\left[V_{r+1}^{+}\right]-\frac{c_{r+1}}{c_{r}} \mathbf{E}\left[V_{r}^{-}\right]\right\} .
$$

The first two sequences go to $P$-a.s. by Lemma (5.9). So we have to prove that

$$
\lim _{r \rightarrow \infty}\left(\frac{c_{r-1}}{c_{r+1}} \mathbf{E}\left[V_{r+1}^{+}\right]-\frac{c_{r+1}}{c_{r}} \mathbf{E}\left[V_{r}^{-}\right]\right)=0 .
$$

Recalling the definitions of $V_{r}^{+}$and $V_{r}^{-}$we can write

$$
\begin{aligned}
& \frac{c_{r-1}}{c_{r+1}} \mathbf{E}\left[V_{r+1}^{+}\right]-\frac{c_{r+1}}{c_{r}} \mathbf{E}\left[V_{r}^{-}\right]=c_{r-1} \sum_{k=1}^{\psi(r+1)-1}(\varphi(k+1)-\varphi(k)) \mathbf{E}\left[Y_{k}^{+}\right]-c_{r+1} \sum_{k=1}^{\psi(r)-1}(\varphi(k+1)-\varphi(k)) \mathbf{E}\left[Y_{k}^{-}\right] \\
& =\left(c_{r-1}-c_{r+1}\right) \sum_{k=1}^{\psi(r+1)-1}(\varphi(k+1)-\varphi(k)) \mathbf{E}\left[Y_{k}^{+}\right]+c_{r+1} \sum_{k=\psi(r)}^{\psi(r+1)-1}(\varphi(k+1)-\varphi(k)) \mathbf{E}\left[Y_{k}^{+}\right]=H_{r}+K_{r},
\end{aligned}
$$

where the second equality holds since the random variables $Y_{n}$ are centered, which gives $\mathbf{E}\left[Y_{n}^{+}\right]=\mathbf{E}\left[Y_{n}^{-}\right]$. For every $n$ we have

$$
\mathbf{E}\left[Y_{n}^{+}\right] \leq \sup \left|g_{1}\right| \leq 2 \sup |f|
$$

From (5.14) we get

$\sum_{k=1}^{\psi(r+1)-1}(\varphi(k+1)-\varphi(k)) \mathbf{E}\left[Y_{k}^{+}\right] \leq 2 \sup |f|\{\varphi(\psi(r+1))-\varphi(1)\} \leq 2 \sup |f| \varphi\left(\varphi^{-1}(r+1)\right)=2 \sup |f|(r+1)$ and (see (5.3))

$$
\sum_{k=\psi(r)}^{\psi(r+1)-1}(\varphi(k+1)-\varphi(k)) \mathbf{E}\left[Y_{k}^{+}\right] \leq 2 \sup |f|\{\varphi(\psi(r+1))-\varphi(\psi(r))\} \leq 4 \sup |f|
$$

From (5.15) and the assumption $\lim _{r \rightarrow \infty}\left(c_{r-1}-c_{r+1}\right) r=0$ we get $\lim _{r \rightarrow \infty} H_{r}=0$, while (5.16) and the assumption $\sum_{n} c_{n}^{2} \log ^{2} n$ (which implies $\lim _{r \rightarrow \infty} c_{r}=0$ ) give $\lim _{r \rightarrow \infty} K_{r}=0$, which concludes the proof of (5.13) and of the Theorem. 
(5.17) ExAmples. We give here some particular cases:

(i) We refer to Example (4.1) (i). Assume that $\gamma_{n}=n^{\beta}$, where $\beta>-1$. Then Theorem (2.9) gives

$$
\lim _{n \rightarrow \infty} \frac{1}{\log n} \sum_{k=1}^{n} \frac{1}{k}\left(1_{A}\left(Z_{k}\right)-P\left(Z_{k} \in A\right)\right)=0 .
$$

On the other hand, in the case $\gamma_{n}=n^{-1}$ we get $\lim _{n \rightarrow \infty} \frac{1}{\log \log n} \sum_{k=1}^{n} \frac{1}{k \log k}\left(1_{A}\left(Z_{k}\right)-P\left(Z_{k} \in A\right)\right)=0$.

(ii) We refer here to Example (4.5). In this case it is easy to see that $\lim _{n \rightarrow \infty} \frac{1}{n} \sum_{k=1}^{n}\left(1_{A}\left(Z_{k}\right)-\right.$ $\left.P\left(Z_{k} \in A\right)\right)=0$.

We conclude this section by proving Remark (2.12). For $\varphi(x)=\log g(x)$ we have $\varphi^{-1}(x)=g^{-1}\left(e^{x}\right)$, hence $G(x)=e^{x}$. Condition (2.11) is verified since

$$
\sum_{k \leq n} \frac{G(k)}{G(n)}+\sum_{k>n} \frac{G(n)}{G(k)}=\sum_{k \leq n} \frac{e^{k}}{e^{n}}+\sum_{k>n} \frac{1}{e^{k-n}}
$$

now the first sum is equal to $\frac{e^{n+1}-1}{e^{n}(e-1)}$, which is bounded as $n \rightarrow \infty$, while the second sum is equal to $\sum_{j} e^{-j}<\infty$.

\section{From the $\vartheta$-dependence coefficient to the Rosenblatt coefficient}

Let $T$ and $S$ be two r. v.'s defined on $(\Omega, \mathcal{A}, P)$. The coefficient of $\vartheta$-dependence $\vartheta(T, S)$ is useful in some cases in order to estimate the Rosenblatt coefficient of dependence of $S$ and $T$, defined as

$$
\alpha(T, S) \doteq \sup _{A, B}\left|\operatorname{Cov}\left(1_{A}(S), 1_{B}(T)\right)\right|,
$$

where the sup is taken over all Borel sets in $\mathbb{R}$.

(6.1) Lemma. Let $A$ be any Borel set in $\mathbb{R}$. Then,

$$
\sup _{f \in \mathcal{L}_{1}}\left|\operatorname{Cov}\left(1_{A}(S), f(T)\right)\right| \leq \vartheta(T, S)
$$

Proof. For any function $f: \mathbb{R}^{d} \rightarrow \mathbb{R}$ such that $f(T)$ is integrable we have

$$
\begin{aligned}
\left|\operatorname{Cov}\left(1_{A}(S), f(T)\right)\right| & =\left|\mathbf{E}\left[1_{A}(S) f(T)\right]-\mathbf{E}\left[1_{A}(S)\right] \mathbf{E}[f(T)]\right| \\
& =\left|\mathbf{E}\left[1_{A}(S) \mathbf{E}[f(T) \mid S]\right]-\mathbf{E}\left[1_{A}(S) \mathbf{E}[f(T)]\right]\right| \\
& =\left|\mathbf{E}\left[1_{A}(S)(\mathbf{E}[f(T) \mid S]-\mathbf{E}[f(T)])\right]\right| \leq \|\left(\mathbf{E}[f(T) \mid S]-\mathbf{E}[f(T)]||_{1} .\right.
\end{aligned}
$$

(6.2) REMARK. If $g$ is $L$-Lipschitzian, since $g / L$ is in $\mathcal{L}_{1}$ we have from (6.1)

$$
\left|\operatorname{Cov}\left(1_{A}(S), g(T)\right)\right|=L\left|\operatorname{Cov}\left(1_{A}(S), \frac{g}{L}(T)\right)\right| \leq L \sup _{f \in \mathcal{L}_{1}}\left|\operatorname{Cov}\left(1_{A}(S), f(T)\right)\right| \leq L \vartheta(T, S) .
$$

(6.3) Proposition. Let $Q_{T}(\epsilon)=\sup _{x} P(x<T \leq x+\epsilon), \epsilon>0$. be the concentration function of $T$. Then for every $x \in \mathbb{R}$,

$$
\left|\operatorname{Cov}\left(1_{A}(S), 1_{(-\infty, x]}(T)\right)\right| \leq \inf _{\epsilon}\left(\frac{1}{\epsilon} \vartheta(T, S)+Q_{T}(\epsilon)\right)
$$

Proof. Fix $\epsilon>0$. Put $g_{\epsilon}(t)=\left(1+\frac{x-t}{\epsilon}\right) 1_{(x, x+\epsilon]}(t)$ and consider the $(1 / \epsilon)$-Lipschitz function $f_{\epsilon}(t)=$ $1_{(-\infty, x]}(t)+g_{\epsilon}(t)$. In view of Remark $(6.2)$, we have

$$
\begin{aligned}
\left|\operatorname{Cov}\left(1_{A}(S), 1_{(-\infty, x]}(T)\right)\right| & =\left|\operatorname{Cov}\left(1_{A}(S), f_{\epsilon}(T)\right)-\operatorname{Cov}\left(1_{A}(S), g_{\epsilon}(T)\right)\right| \\
& \leq\left|\operatorname{Cov}\left(1_{A}(S), f_{\epsilon}(T)\right)\right|+\left|\operatorname{Cov}\left(1_{A}(S), g_{\epsilon}(T)\right)\right| \leq \frac{1}{\epsilon} \vartheta(T, S)+Q_{T}(\epsilon) .
\end{aligned}
$$

Since $\epsilon>0$ is arbitrary, the proof is achieved. 
We now consider a case in which the infimum in (6.4) can be explicitly calculated. Assume that $T$ is such that, for some fixed $\gamma>0$ and for every $\epsilon>0$,

$$
Q_{T}(\epsilon) \leq C_{1} \epsilon^{\gamma}
$$

(6.6) Proposition. We have

$$
\sup _{A, x}\left|\operatorname{Cov}\left(1_{A}(S), 1_{(-\infty, x]}(T)\right)\right| \leq C_{2}(\gamma)(\vartheta(T, S))^{\gamma /(\gamma+1)}
$$

Proof. We introduce the bound of $Q_{T}$ given in (6.5) into the infimum in (6.4). Then such infimum can be found by means of elementary calculus. It is attained for $\epsilon=\left(\vartheta(T, S) / C_{1}\right)^{1 /(\gamma+1)}$, proving (6.7).

(6.8) Remark. Assumption (6.5) is simply $\gamma$-holderianity of the distribution function of $T$, since

$$
P(x<T \leq x+\epsilon)=F_{T}(x+\epsilon)-F_{T}(x) .
$$

If the law of $T$ has a bounded density, then (6.5) holds with $\gamma=1$. A uniform version of (6.5) for a sequence of random variables is considered in the paper [3] (formula (2.9) pag. 317).

We now discuss another relevant case in which $(6.5)$ holds. Let $\left(X_{n}\right)$ be a sequence of independent identically distributed random variables with distribution belonging to the domain of attraction of a stable distribution $\Phi$ with exponent $\alpha \in(1,2]$ (see section 4, example (4.1) (iii)). Put, as in section 4, $Z_{n}=\frac{S_{n}}{a_{n}}$. For $p<q$ we consider $T=Z_{q}$ and $S=Z_{p}$, so that our task is to bound the concentration function of $Z_{q}$. This can be done by using the following result (see [10], pag 68 for the proof).

(6.9) Lemma. Let $\left(X_{n}\right)$ be a sequence of independent random variables and put $S_{n}=X_{1}+\cdots+X_{n}$. Let $\lambda_{1}, \lambda_{2}, \ldots, \lambda_{n}$ be positive numbers such that $\lambda_{k} \leq \lambda, k=1, \ldots, n$. Let $\left(X_{k}^{s}\right)$ denote a symmetrized version of $\left(X_{k}\right)$. Then

$$
Q_{S_{n}}(\lambda) \leq C_{3} \lambda\left(\sum_{k=1}^{n} \lambda_{k} 2 P\left\{\left|X_{k}^{s}\right| \geq\left(\lambda_{k} / 2\right)\right\}\right)^{-1 / 2}
$$

For each $k=1, \ldots, q$ take $\lambda_{k}=\lambda=a_{q} \epsilon$ in (6.10). We get

$$
\begin{aligned}
Q_{Z_{q}}(\epsilon) & =Q_{S_{q}}\left(a_{q} \epsilon\right) \leq C_{3} a_{q} \epsilon\left(a_{q}^{2} \epsilon^{2} q\left(1-F\left(a_{q}(\epsilon / 2)\right)+F\left(-a_{q}(\epsilon / 2)\right)\right)\right)^{-1 / 2} \\
& =C_{3}\left(q\left(1-F\left(a_{q}(\epsilon / 2)\right)+F\left(-a_{q}(\epsilon / 2)\right)\right)\right)^{-1 / 2} .
\end{aligned}
$$

By formulas (5.6) and (5.9) p. 575 of [5] we know that $\lambda^{\alpha} q\left(1-F\left(a_{q} \lambda\right)+F\left(-a_{q} \lambda\right)\right) \rightarrow C_{4}>0$. Hence, for large $q$ we obtain $Q_{Z_{q}}(\epsilon) \leq C_{5} \epsilon^{\alpha / 2}$.

In [6] the following statement is proved:

(6.11) Proposition. For large $q$ and every $p \leq q$ we have

$$
\sup _{A, x}\left|\operatorname{Cov}\left(1_{A}\left(Z_{p}\right), 1_{(-\infty, x]}\left(Z_{q}\right)\right)\right| \leq C_{\alpha}\left(\frac{a_{p}}{a_{q}}\right)^{\alpha /(\alpha+2)}
$$

It is also a consequence of Proposition (6.6) and formula (4.2) above. 


\section{References}

[1] Berkes I., Csáki E. (2001) A universal result in almost sure central limit theory, Stoch. Proc. Appl. 94, 105-134.

[2] Dedecker J., Doukhan P. (2003) A new covariance inequality and applications, Stoch. Proc. Appl. 106, 63-80.

[3] Doukhan P., Louhichi S. (1999) A new weak dependence condition and applications to moment inequalities, Stoch. Proc. Appl. 84, 313-342.

[4] Duflo M. (1997) Random Iterative Models, Springer.

[5] Feller W. (1971) An Introduction to Probability Theory and Its Applications, Wiley.

[6] Giuliano-Antonini R., Weber M. (2005) Counting Occurrences in Almost Sure Limit Theorems, Coll. Math. 102, no. 2, 271-289.

[7] Lacey M., Philipp W. (1990) A note on the almost sure central limit theorem, Stat. \& Prob. Letters 9, 201-205.

[8] Móri T. F., Székely B. (2003) Almost sure convergence of weighted partial sums, Acta Math. Hungar. 99 (4), 285-303.

[9] Olevskii A. M. (1975) Fourier series with respect to general orthogonal systems, Ergebnisse der Mathematik und ihrer Grenzgebiete, Band $\mathbf{8 6 .}$

[10] Petrov V. V. (1995) Limit Theorems of Probability Theory, Oxford Science Publications.

[11] Weber M. (1998) Entropie métrique et convergence presque partout, Coll. "Travaux en Cours", 58, Hermannn, Paris.

Rita Giuliano-Antonini: [Corresponding author] Dipartimento di Matematica, Università di Pisa, Largo B. Pontecorvo, 5, 56100 Pisa, Italy. EmAIL: giuliano@dm.unipi.it

Michel WeBer: U.F.R. de Mathématique (IRMA), Université Louis-Pasteur et C.N.R.S., 7 rue René Descartes, F-67084 Strasbourg Cedex. EmAIL: weber@math.u-strasbg.fr 